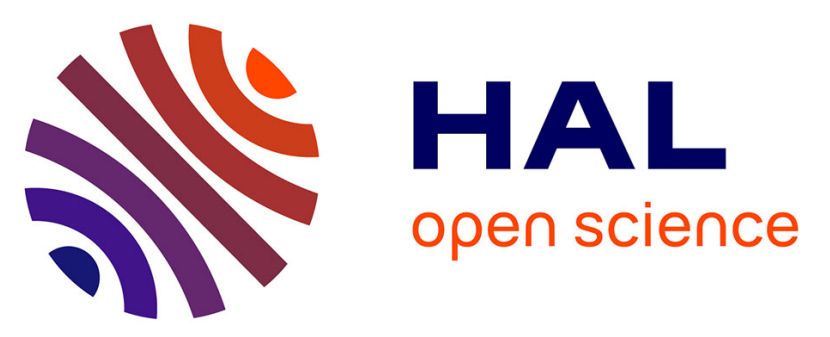

\title{
What do stirred yogurt microgels look like? Comparison of laser diffraction, 2D dynamic image analysis and 3D reconstruction
}

Marine Moussier, Valérie Guenard-Lampron, Kevin Lachin, Gabrielle Moulin, Sylvie L. Turgeon, Camille Michon, Delphine Huc-Mathis, Véronique Bosc

\section{To cite this version:}

Marine Moussier, Valérie Guenard-Lampron, Kevin Lachin, Gabrielle Moulin, Sylvie L. Turgeon, et al.. What do stirred yogurt microgels look like? Comparison of laser diffraction, 2D dynamic image analysis and 3D reconstruction. Food Structure, 2019, 20, 10.1016/j.foostr.2019.100107 . hal-02620143

\section{HAL Id: hal-02620143 \\ https://hal.inrae.fr/hal-02620143}

Submitted on 22 Oct 2021

HAL is a multi-disciplinary open access archive for the deposit and dissemination of scientific research documents, whether they are published or not. The documents may come from teaching and research institutions in France or abroad, or from public or private research centers.
L'archive ouverte pluridisciplinaire HAL, est destinée au dépôt et à la diffusion de documents scientifiques de niveau recherche, publiés ou non, émanant des établissements d'enseignement et de recherche français ou étrangers, des laboratoires publics ou privés.

\section{(c) (1) $\$$}

Distributed under a Creative Commons Attribution - NonCommerciall 4.0 International 


\title{
What do stirred yogurt microgels look like? Comparison of laser diffraction, 2D dynamic image analysis and 3D reconstruction
}

\author{
Marine Moussier ${ }^{\mathrm{a}}$, Valérie Guénard-Lampron ${ }^{\mathrm{b}}$, Kevin Lachina, Gabrielle Moulin ${ }^{\mathrm{a}}$, Sylvie L. \\ Turgeon $^{\mathrm{b}}$, Camille Michon ${ }^{\mathrm{a}}$, Delphine Huc-Mathis ${ }^{\mathrm{a}}$, Véronique Bosc ${ }^{\mathrm{a}}$ \\ ${ }^{a}$ Ingénierie Procédés Aliments, AgroParisTech, Inra, Université Paris-Saclay, 91300 Massy, France \\ ${ }^{b}$ Dairy Science and Technology Research Centre (STELA), Institute of Nutrition and Functional Foods (INAF), \\ Université Laval, Quebec City, Qc, G1V 0A6, Canada
}

\section{Graphical abstract}

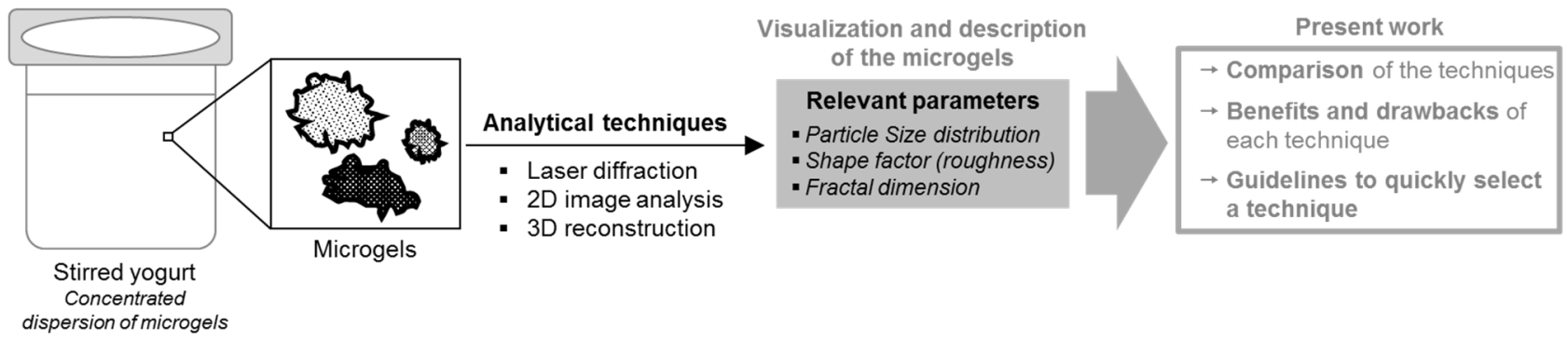

\section{Highlights}

- Microgel size distributions were similar using LD, 2D and 3D techniques.

- Microgels were non-spherical, rough and heterogeneous with the 2D and 3D analyses.

- Estimations of the fractal dimension were more reliable using LD and 3D than 2D.

- LD was relevant, fast and versatile in accessing size and fractal dimension.

- 2D was faster and 3D more accurate in accessing both shape and fractal dimension.

\section{Abstract}

Stirred yogurts can be considered as concentrated dispersions of microgels. The size, shape and fractal dimension of these microgels are known to have a direct impact on textural and sensory properties of stirred yogurts, consequently their thorough characterization is of interest. Different techniques can be used including laser diffraction (LD), 2D dynamic image analysis or 3D reconstruction from z-stack confocal images. The aim of this study was to compare the ability of the three techniques to describe the size, shape and fractal dimension of the stirred yogurt microgels. Two stirred yogurts with different compositions, one fat free $(0.1 \%)$ and one high fat $(9.3 \%)$, were used. The microgel size distributions obtained were similar using LD, 2D image analysis and 3D reconstruction. Additionally, 2D image analysis and 3D reconstruction enabled visualization of the microgels and access to their shape through morphological factors such as roughness index. The microgels observed were non-spherical, rough and heterogeneous in shape. All three techniques also made it possible to determine the fractal dimension of the microgels, but 2D image analysis displayed lower values than $\mathrm{LD}$ and $3 \mathrm{D}$ reconstruction.

\section{Keywords}

Size, Shape; Stirred yogurt microgels; Laser diffraction; 2D dynamic image analysis; 3D reconstruction. 


\section{Introduction}

From a structural point of view, stirred yogurts are concentrated dispersions of microgels (soft particles) whose diameters range from 10 to $100 \mu \mathrm{m}$ (Sodini, Remeuf, Haddad, \& Corrieu, 2004; Van Marle, 1998). In presence of fat, each microgel can itself be considered as an emulsion-filled gel, with fat dispersed as droplets that interact with the protein network via the interface located on the surface of fat globules and mainly composed of milk proteins. The term "microgels" is used for the entities obtained after the set yogurt is stirred. Structurally speaking, these microgels are "aggregates" of primary particles of fat droplets and proteins (mainly whey protein / casein micelle complexes). It is established that stirring causes profound changes in the textural and sensory properties of the yogurts by breaking the continuous gel (i.e. set yogurt) into microgels (soft particles) (Cayot, Schenker, Houzé, Sulmont-Rossé, \& Colas, 2008; Lee \& Lucey, 2006). (Shewan \& Stokes, 2013) have also demonstrated that the properties of soft particle concentrated dispersions are directly impacted by the properties of the dispersed particles (microgels in the case of stirred yogurts): their hardness, size distribution or shape. Having access to reliable data on particle size distribution (PSD), the distributions of shape factors and average mass fractal dimension of the microgels is thus of interest to understand the textural properties of stirred yogurts.

Laser diffraction (LD) particle size analysis (or static light scattering) is commonly used to access the size distribution of stirred yogurt microgels (Chung, Degner, \& Julian, 2014; Hahn, Sramek, Nöbel, \& Hinrichs, 2012; Huc, Michon, Bedoussac, \& Bosc, 2016; Nöbel et al., 2016). This technique measures particles ranging from 0.02 to $2,000 \mu \mathrm{m}$ in diameter. To do so, a laser beam of known wavelength $(\lambda=633$ $\mathrm{nm}$ ) irradiates the suspension to be analyzed, and detectors located at specific angles collect the intensity of the light scattered by the particles. Assuming spherical particles with homogenous composition, the software then uses the Mie theory to deduce a theoretical PSD from the light scattering results obtained with LD. To successfully use the Mie theory, knowledge of the refractive and absorbance indexes of the dispersed medium is required (Malvern Instruments Ltd., 2007). In the specific case of the stirred yogurt microgels, these optical indexes are difficult to access. The question of the consistency of the LD size measurement of complex systems like stirred yogurt microgels thus naturally arises. As this technique does not enable access to shape, other techniques of image analysis can be used, compared and possibly combined with LD to obtain the most accurate results possible.

2D dynamic image analysis is a recently developed technique that enables precise access to the PSD and to the shape of different types of particles (Carugo et al., 2015; Mallipeddi, Saripella, \& Neau, 2014; Perez et al., 2017). This technique can be compared to a modern microscope using a pulsed light source and a high speed mega-pixel camera (Köhler, Stübinger, List, \& Witt, 2008; List, Köhler, Witt, Gmbh, \& Pulverhaus, 2011). Unlike laser diffraction analysis, image analysis directly records the properties of the image of each particle to determine diameter and shape factors. 2D image analysis thus appears to be an appropriate tool to access data concerning the morphology of stirred yogurt microgels. Many shape factors are described in the literature for non-spherical microgels (convexity, roundness, circularity, sphericity or roughness) and the definitions of these factors depends on the equipment and analytical technique used 
(Hentschel \& Page, 2003; Podczek, 1997; Yan \& Su, 2017). However, the roughness index was the most often used, because it is relevant regarding surface heterogeneity.

A variety of microscopic techniques are used to assess the microstructure of stirred yogurts (Mortazavian, Rezaei, \& Sohrabvandi, 2009). These include transmission electron microscopy (TEM), scanning electron microscopy (SEM) and confocal laser scanning microscopy (CLSM), sometimes associated with image analysis (Torres, Amigo Rubio, \& Ipsen, 2012). Both TEM and SEM are high resolution techniques, but sample preparation can be complex and quite expensive. Moreover, structure artefacts due to the sample preparation are very often suspected. CLSM is a low-invasive alternative requiring the staining of the compounds to be observed. In particular, this technique makes it possible to obtain a series of two-dimensional images $(\mathrm{x}, \mathrm{y})$ by $\mathrm{z}$-stacking. Using the appropriate software, these images can be compiled and computed into a 3D representation. This technique was recently applied to food systems such as continuous model gels (whey protein isolate/polysaccharide) (van den Berg et al., 2008) or soft apple cells (Leverrier, Moulin, Cuvelier, \& Almeida, 2017). However, to the best of our knowledge, CLSM associated with 3D reconstruction has not yet been used to assess the size and shape of the microgels of stirred yogurts.

Laser diffraction, 2D dynamic image analysis and 3D reconstruction can also be used to extract information on the structure of the samples by means of the mass fractal dimension $\left(D_{f}\right)$. This structural parameter is closely linked to the concept of fractal geometry (object having a structure independent of the scale of observation) and thus compactness. Fractal geometries were first mathematically introduced by Mandelbrot (1975) in the mid-1970s and later used in the field of colloid and aggregates, thus opening a new way of characterizing the structure of aggregates in terms of occupancy rate and compaction of the structure in the volume of the aggregates (Andoyo, Guyomarc, \& Burel, 2015; Mellema, Walstra, van Opheusden, \& van Vliet, 2002) or roughness and sphericity of the aggregates (Raper \& Amal, 1993; Torres et al., 2012). It has been accepted for many years (Forrest \& Witten, 1979) that aggregates can be described as fractal-like structures, meaning their mass scales with a characteristic radius through the use a specific dimension named the mass fractal dimension. Unlike the topological dimension, which is stricly an integer (between 1 and 3), the fractal dimension is usually a non-integer number. The use of accurate fractal dimensions thus makes it possible to replace the conventional sphericity assumptions that can be used in modelling the relationship between structural and textural properties.

As LD measurements are the most widely used in the dairy field, but have limitations, the first objective of this study was to analyze its suitability for heterogeneous (in composition) and irregularly shaped systems like microgels. This analyze of reliability was made by comparing the LD results with the ones obtained with 2D image analysis and 3D reconstruction (from confocal images). The other objective was to compare the ability of the three different techniques to provide information on the size, shape and fractal dimension of stirred yogurt microgels. For this purpose, a fat free and a high fat commercial yogurts were selected and diluted in purified water. The size distributions and the fractal dimensions of the microgels were determined using all three techniques, whereas their shape factors (length and roughness index 
distributions) were determined only using 2D and 3D image analyses. The different results obtained were then compared and analyzed as a function of the technique.

\section{Materials and methods}

\subsection{Stirred yogurt sampling}

Two types of plain stirred yogurts from different commercial brands were purchased in the market. Perle de Lait (Yoplait, France) was chosen for its high fat (F) content and its classic protein (P) content (9.3 $\mathrm{g} / 100 \mathrm{~g}$ fat, $3.2 \mathrm{~g} / 100 \mathrm{~g}$ protein). Taillefine Le Brassé 0\% (Danone, France) was selected because it is fat free and has a quite high protein content $(0.1 \mathrm{~g} / 100 \mathrm{~g}$ fat, $4.5 \mathrm{~g} / 100 \mathrm{~g}$ protein). For the rest of the study, the stirred yogurt samples are referred as FP3 for Perle de Lait and P4.5 for Taillefine Le Brassé 0\%. FP3 and P4.5 were chosen to have a similar aging time (based on their similar expiration dates). They were stored in the same conditions (i.e. at $4{ }^{\circ} \mathrm{C}$ ). All the measurements were performed on two consecutive days. Purified water used for the dilutions was obtained using a Milli-Q purification system (Millipore, Merck, Germany). It was checked and proved that the level of dilution did not have a significant impact on the results. To achieve good sampling and homogeneity, each yogurt was gently mixed using a small spoon rotated 4 times from the bottom of the pot towards the top, with a quarter turn between each movement. For this study, three dilutions were performed from different pots of a same batch of FP3 and of P4.5.

\subsection{Laser diffraction analysis}

Stirred yogurts were diluted 1:10 $(w / w)$ with purified water in a $100 \mathrm{~mL}$ pot and the microgels were dispersed by reversing the pot several times. Size distributions were measured by laser diffraction with a MasterSizer 2000 (Malvern Instruments, UK). To achieve a constant level of obscuration, only some drops of 1:10 diluted stirred yogurts were poured in dispersant tank for the measurement (three repetitions), resulting in a total dilution of 1:100. A refractive index of 1.33 for water and 1.46 for the microgels (refractive index of milk proteins), and an absorption index of 0.01 for the microgels were used (Huc et al., 2016). Several data were deduced from the PSD (Malvern Instruments Ltd., 2007): size volume distribution, particle sizes representing less than $10 \%(d(0.1), \mu \mathrm{m}), 50 \%$ (median diameter $d(0.5), \mu \mathrm{m})$ and $90 \%(d(0.9)$, $\mu \mathrm{m})$ of the sample, volume $(D[4,3], \mu \mathrm{m})$ and surface (Sauter mean diameter $D[3,2], \mu \mathrm{m})$ weighted mean diameters ( $D[m, n]$, Eq. 1$)$ and width of the distribution (span, Eq. 2).

$$
\begin{gathered}
D[m, n]=\left[\frac{\sum \text { volume }_{i} \times d_{i}{ }^{m-3}}{\sum \text { volume }_{i} \times d_{i}{ }^{n-3}}\right]^{\frac{1}{m-n}} \\
\text { span }=\frac{d(0.9)-d(0.1)}{d(0.5)}
\end{gathered}
$$

In addition, it was also possible to extract the fractal dimension of the microgel aggregates from the scattering data. The light scattered by porous aggregated structures entails more modeling complexity than 
135

136

137

138

139

140

141

the scattering of solid homogenous spheres. One way to overcome this problem is to use the Rayleigh-GansDebye theory (Gregory, 2009; Sorensen, 2001). Assuming the primary particles that comprise the aggregate behave like Rayleigh scatterers (i.e. the diameter of the initial particles is much smaller than the wavelength of the incident beam $\lambda$ ), it is possible to introduce a structure factor $S(q)$ in the expression of the light scattered intensity $I(q)$ so that (Eq. 3):

$$
I(q) \propto S(q) * P(q)
$$

Eq. 3

where $P(q)$ is the form factor and is due to primary particles. $q\left(\mathrm{~m}^{-1}\right)$ is the scattering vector and is expressed by Eq. 4 , where $\theta$ is the scattering angle and $n$ the refractive index of the dispersing medium.

$$
q=4 \pi \frac{n}{\lambda} \sin \left(\frac{\theta}{2}\right)
$$

As $q^{-1}$ represents the characteristic length probed with the light scattering measurement, information on the aggregate structure can only reasonably be extracted for $q^{-1}$ values so that $r_{0}<<q^{-1}<<R_{a g}$, where $r_{0}$ denotes the characteristic size of the primary particles, and $R_{a g}(\mathrm{~m})$ the characteristic size of the aggregates. Under this condition, the structure factor depends on the fractal dimension, and it is thus possible to write the proportionality relation between the intensity of the light scattered and the structure factor as stated by Eq. 5 .

$$
I(q) \propto q^{-D_{f}}
$$

Eq. 5

Using a Log-Log scale plot, it was thus possible to access the mean mass fractal dimension of the sample by simply determining the slope of the scattering plot in the above-mentionned $q^{-1}$ region (see Fig. 5 in Supplementary material). This theory has been successfully applied in several studies involving colloidal suspensions, particularly latexes, well calibrated in size and shape (Burns, Yan, Jameson, \& Biggs, 1997; Lachin et al., 2017; Selomulya, Amal, Bushell, \& Waite, 2001). More closely connected with the food and dairy industries, some successes have been achieved in the light scattering study of model casein and micellar casein aggregates (Chardot, Banon, Misiuwianiec, \& Hardy, 2002; Panouillé, Durand, Nicolai, Larquet, \& Boisset, 2005; Vétier, Banon, Chardot, \& Hardy, 2003).

\subsection{D dynamic image analysis}

Dynamic image analysis was performed using a QICPIC/R modular particle size and shape analyzer and a LIXELL wet dispersing unit (Sympatec GmbH, DE). A precision M4 lens measuring from 1 to $750 \mu \mathrm{m}$ with a $0.5 \mathrm{~mm}$ cuvette was used. Stirred yogurts were diluted 1:2000 (w/w) with purified water in a $1000 \mathrm{~mL}$ beaker to disperse the microgels and the dispersed microgels were then stirred at $100 \mathrm{rpm}$ for $1 \mathrm{~min}$ and pumped into the dispersing unit with a peristaltic pump (Masterflex L/S Model 77201-60, Cole-Parmer, FR) at a flow rate of $25 \mathrm{~mL} / \mathrm{min}$. For each dilution, two 30-second image acquisitions were performed at $10 \mathrm{~Hz}$. The images were processed using PAQXOS application software (PAQXOS, Version 2.2.2, Sympatec $\mathrm{GmbH}, \mathrm{DE})$. Size measurement data such as volume distribution, $d(0.1), d(0.5), d(0.9), D[4,3]$ and $D[3,2]$ were retrieved from the image analysis. The diameters of the equivalent surface circle of microgels and maximum $\left(F_{\max }, \mu \mathrm{m}\right)$ and minimum $\left(F_{\min }, \mu \mathrm{m}\right)$ Feret diameters, derived respectively using the maximum and 
minimum distance between two tangents of the contour of the particle, were determined by the software. The width of the distribution (span) was calculated by Eq. 2. The software was also able to determine shape factors including the roughness index (Eq. 6).

$$
\text { roughness }(2 D)=\frac{\text { perimeter of equivalent circle }}{\text { real perimeter }}
$$

Eq. 6

The results of the dynamic image analysis made it possible to measure fractal dimensions. Some studies have already proposed methods of calculating two-dimensional fractal dimensions $\left(\mathrm{D}_{2}\right)$ from image analysis (Jiang \& Logan, 1991; Serra \& Casamitjana, 1998). The two-dimensional fractal dimension was determined by the relationship between the area $(A)$ of the microgels and their maximum Feret diameter $\left(F_{\max }\right)(\mathrm{Eq} .7)$. In the specific case of the calculation of the two-dimensional fractal dimensions, the microgels below $10 \mu \mathrm{m}$ in diameter were not selected due to their low image resolution $(1 \mu \mathrm{m}=1$ pixel $)$. For each yogurt analysis, 7,000 microgel images were randomly selected and classified according to their roughness index. For each class of roughness, a plot $\log (A)$ vs. $\log \left(F_{\max }\right)$ was performed. A weighted average of these classes was performed to determine a representative $\mathrm{D}_{2}$ value of all measured stirred yogurts.

$$
A \propto F \max ^{D_{2}}
$$

Eq. 7

Using simulated aggregates, Lee \& Kramer (2004) found a relationship between the two-dimensional fractal dimension $\left(D_{2}\right)$ obtained from image analysis and the three-dimensional fractal dimension $\left(D_{3}\right)$ from the laser diffraction results (Eq. 8). The equation was validated by comparing experimental $D_{3}$ (laser diffraction and electrical sensing) with simulated $D_{3}$ on different particles, particularly spherical ones (Baalousha, Manciulea, Cumberland, Kendall, \& Lead, 2008; Lee \& Kramer, 2004).

$$
D_{3}=1.391+0.01 e^{2.164 D_{2}}
$$

Eq. 8

\subsection{D reconstruction from confocal images}

\subsubsection{Acquisition by confocal microscopy and 3D processing}

The stirred yogurts were first diluted 1:100 $(w / w)$ with purified water in a $100 \mathrm{~mL}$ pot, and the microgels were then gently dispersed by reversing the pot several times. The proteins that made up the microgels were then stained by mixing $250 \mu \mathrm{L}$ of this solution with $2.5 \mu \mathrm{L}$ of DyLight $488 \mathrm{~nm}$ (Thermo Fisher Scientific, Waltham, MA, USA) (one repetition per dilution). Confocal images were acquired with a TCS SP8 AOBS inversed confocal laser scanning microscope (CLSM) (Leica, Solms, Germany) equipped with a Helium-Neon laser (458 nm excitation wavelength) and an Argon laser (633 nm excitation wavelength). From 93 to 195 images $(\mathrm{x}, \mathrm{y})$ were acquired by z-scan $(0.8 \mu \mathrm{m}$ steps) with a magnification $\times 40$. For each sample, the z-stacks obtained were combined and processed to reconstitute the 3D microgels using Scan IPTM software (version 7.0, build 2656, () 2000-2014 Simpleware Ltd.). The different processing steps are based on the work of Leverrier et al. (2017) and are illustrated in Fig. 1. The 2D confocal images (x, y) of 
each z-series were first combined into a 3D reconstruction. A median filter was then applied to the background of the images (neighborhood radius of $1 \times 1 \times 1$ pixel) to eliminate noise. By comparison with the initial confocal images, a threshold was eventually chosen to select the level of grey that differentiated the stained microgels from the background. The 3D reconstitutions shown here were chosen as being representative of the replications.

1

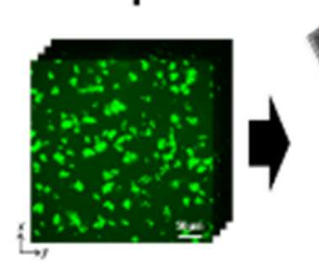

2

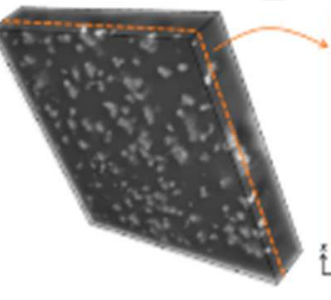



a



3



Fig. 1 Processing steps used to reconstitute the 3D microgels of stirred yogurt: 1) z-acquisition of 2D confocal images (x, y) (proteins in green); 2) 3D reconstruction and application of a median filter $(\mathbf{a} \rightarrow \mathbf{b})$; 3) 3D identification of the microgels.

202

203

204

205

206

207

208

209

210

211

212

213

214

215

216

\subsubsection{Data computation from $3 D$ reconstruction}

From the 3D reconstructions, Scan $\mathrm{IP}^{\mathrm{TM}}$ software provided several data on both size and shape, some of which were either recovered or processed in this study. First, the software provided the number of individual microgels identified in the 3D reconstruction and their corresponding volume $\left(\mu \mathrm{m}^{3}\right)$. In order to obtain the size distribution of the microgels in equivalent sphere, their volumes were discretized (logarithmic scale). To ensure good quality discretization, at least eight classes were required to plot each distribution (i.e. with volume fractions greater than $0 \%$ ), with a minimum of two classes per decade. Like with laser diffraction, $d(0.1)(\mu \mathrm{m}), d(0.5)$ (median diameter, $\mu \mathrm{m}), d(0.9)(\mu \mathrm{m}), D[3,2], D[4,3]$ and span were retrieved from the reconstituted size distribution. From the volume of each microgel, their equivalent sphere diameter (Eq. 9) then the surface of their equivalent sphere (Eq. 10) were calculated. A roughness index of the microgels was calculated by dividing the surface of an equivalent sphere by the real surface $\left(\mu \mathrm{m}^{2}\right)$ given by the software (Eq. 11). For each microgel, the volumes of the oriented bounding ellipsoid and the corresponding minor, medial and major lengths $(\mu \mathrm{m})$ were also obtained using the same software. The distributions of the roughness index, minor length and major length were plotted by discretizing the data (using the volume of equivalent sphere).

$$
\begin{gathered}
\text { equivalent sphere diameter }(\mu m)=\sqrt[3]{\frac{6 \times \text { volume }}{\pi}} \\
\text { surface of equivalent sphere }\left(\mu m^{2}\right)=\pi \times\left(\sqrt[3]{\frac{6 \times \text { volume }}{\pi}}\right)^{2} \\
\text { roughness }(3 D)=\frac{\text { surface of equivalent sphere }}{\text { real surface }}
\end{gathered}
$$


It was also possible to use the data provided by the 3D processing to estimate the mean fractal dimension of the sample concerned. By definition of the fractal scaling, the mass of a fractal aggregate $m_{a g}$ $(\mathrm{kg})$ composed of initial particles of radius $r_{0}(\mathrm{~m})$ and mass $m_{0}(\mathrm{~kg})$ can be linked to the characteristic cluster size $R_{a g}$ so that (Bushell, Yan, Woodfield, Raper, \& Amal, 2002; Gregory, 2009; Lazzari, Nicoud, Jaquet, Lattuada, \& Morbidelli, 2016) (Eq. 12):

$$
n_{p}=\frac{m_{a g}}{m_{0}}=k_{0} \cdot\left(\frac{R_{a g}}{r_{0}}\right)^{D_{f}}
$$

where $n_{p}$ stands for the original number of particles in the aggregate. The radius of gyration is often taken as the characteristic aggregate size. However, as mentioned by Lazzari et al. (2016), any characteristic length of the aggregate can be used instead. The shape of the relation remains identical, but the effective value of $k_{0}$ changes. The effective density $\rho_{e}$ of a fractal aggregate (taking its porosity into account) is proportional to $R_{a g}$ as presented by Eq. 13 (Gregory, 2009):

$$
\rho_{e} \propto R_{a g}^{3-D_{f}}
$$

The 3D reconstruction did not allow the determination of the mass of each single aggregate. However, it provided values for the volume of each aggregate $V_{a g}\left(\mathrm{~m}^{3}\right)$ and its surface envelope $S_{a g}\left(\mathrm{~m}^{2}\right)$. In this study, it was chosen to use the ratio $V_{a g} / S_{a g}$ as the characteristic length of the aggregates. By combining the two last mentioned relations (Eq. 12 and Eq. 13), it was then possible to find a proportionality relation between the volume of the aggregates and its volume-over-surface ratio so that (Eq. 14):

$$
V_{a g} \propto\left(\frac{V_{a g}}{S_{a g}}\right)^{D_{f} / 3-D_{f}}
$$

Eq. 14

Thus, by plotting $\log \left(V_{a g}\right)$ vs. $\log \left(V_{a g} / S_{a g}\right)$ (see Fig. 5 in Supplementary material) for all the stirred yogurt microgels, and extracting the slope of the linear correlation, it was possible to estimate the average mass fractal dimension of the microgels.

\subsection{Statistical analysis}

Statistical analyses were performed using XLSTAT 2015.1 software (Addinsoft, Paris, France). Analysis of variance (ANOVA) was used to evaluate differences between values using Tuckey's test. A significance level of $p<0.05$ was used.

\section{Results and discussion}

The size distribution, shape and fractal dimension of the stirred yogurt microgels were measured using the three techniques (laser diffraction and/or 2D image analysis and 3D reconstruction) and are reported in the following tables and figures in order to evaluate the suitability, advantages and limitations of the three techniques. The two stirred yogurts (FP3 and P4.5) are rarely compared since they are intentionally chosen as being different to compare techniques in two systems representative of the variety of stirred yogurt microstructures. 


\subsection{Comparison of microgel size distributions (LD, 2D, 3D)}

Fig. 2 shows the size distributions obtained using the three measurement techniques and, below, some data that are characteristic of these distributions. For both FP3 (Fig. 2 A) and P4.5 stirred yogurts (Fig. 2 B), the distributions obtained by laser diffraction, 2D image analysis and 3D reconstruction were all unimodal and rather overlapped for a given stirred yogurt. The FP3 microgels were smaller than those of the P4.5 stirred yogurt, with a median size between 10 and $16 \mu \mathrm{m}$ for FP3 and between 17 and $24 \mu \mathrm{m}$ for P4.5. These results mainly indicate that the three techniques are consistent. Moreover, the orders of magnitude of the obtained sizes are in accordance with measurements made by some authors who used laser diffraction or CLSM for different stirred yogurts (Cayot et al., 2008; Hahn et al., 2015; Huc et al., 2016). The differences between the two stirred yogurts (Fig. 2 A and B) were certainly mainly due to their composition and their stirring process, which are known to have the most impact on microgel size (Mokoonlall, Nöbel, \& Hinrichs, 2016; van Marle, van den Ende, de Kruif, \& Mellema, 1999).

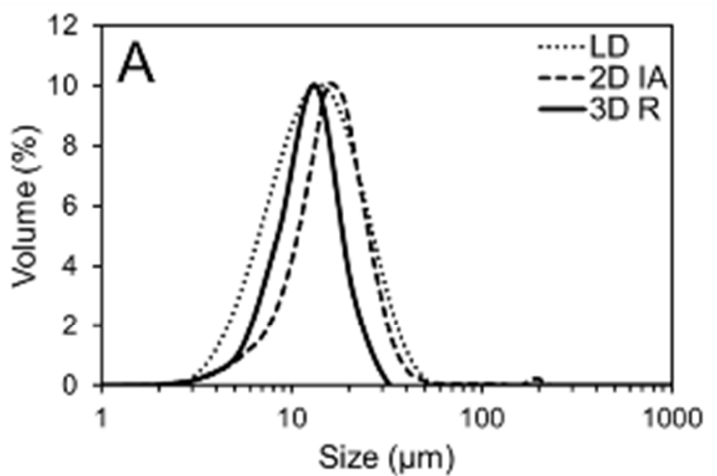

\begin{tabular}{|cccc|}
\hline & LD $^{1}$ & 2D IA2 & $3 \mathrm{D} \mathrm{R} \mathrm{R}^{3}$ \\
\hline Particle number & - & $>500000$ & $254-379$ \\
$\mathrm{D}[3.2](\mu \mathrm{m})$ & $12.1 \pm 0.9^{\circ} \mathrm{b}$ & $13.6 \pm 1.4^{\circ \circ}$ & $11.2 \pm 0.7^{\circ}$ \\
$\mathrm{D}[4,3](\mu \mathrm{m})$ & $16.1 \pm 1.2^{\circ}$ & $18.0 \pm 0.7^{\circ}$ & $13.1 \pm 1.2^{\circ}$ \\
$\mathrm{d}(0.1)(\mu \mathrm{m})$ & $6.8 \pm 0.5^{\circ}$ & $8.5 \pm 1.1^{\circ}$ & $5.9 \pm 0.4^{\circ}$ \\
$\mathrm{d}(0.5)(\mu \mathrm{m})$ & $14.3 \pm 1.1^{\circ}$ & $16.2 \pm 1.5^{\circ}$ & $10.5 \pm 0.8^{\circ}$ \\
$\mathrm{d}(0.9)(\mu \mathrm{m})$ & $28.0 \pm 2.0^{\circ}$ & $26.6 \pm 2.2^{\circ}$ & $15.9 \pm 2.5^{\circ}$ \\
Span & $1.5 \pm 0.04^{\circ}$ & $1.1 \pm 0.0^{\circ}$ & $0.9 \pm 0.2^{\circ}$ \\
\hline
\end{tabular}



\begin{tabular}{|c|c|c|c|}
\hline & LD1 $^{1}$ & $2 \mathrm{D} \mathrm{IA}^{2}$ & $3 \mathrm{D} \mathrm{R}^{3}$ \\
\hline Particle number & - & $>500000$ & $67-233$ \\
\hline $\mathrm{D}[3,2](\mu \mathrm{m})$ & $18.6 \pm 0.3^{a . b}$ & $19.2 \pm 0.7=$ & $17.8 \pm 1.1$ \\
\hline $\mathrm{D}[4,3](\mu \mathrm{m})$ & $25.4 \pm 0.4^{b}$ & $27.5 \pm 1.0^{\circ}$ & $21.8 \pm 20^{\circ}$ \\
\hline $\mathrm{d}(0.1)(\mu \mathrm{m})$ & $10.3 \pm 0.2^{b}$ & $11.1 \pm 0.3^{\circ}$ & $9.3 \pm 0.7^{c}$ \\
\hline $\mathrm{d}(0.5)(\mu \mathrm{m})$ & $22.2 \pm 0.3^{b}$ & $23.7 \pm 1.2^{y}$ & $16.9 \pm 1.2$ \\
\hline$d(0.9)(\mu \mathrm{m})$ & $45.1 \pm 0.8^{\circ}$ & $48.4 \pm 1.6^{n}$ & $27.9 \pm 3.2^{\circ}$ \\
\hline Span & $1.6 \pm 0.04^{\circ}$ & $1.6 \pm 0.0^{\circ}$ & $1.1 \pm 0.1^{b}$ \\
\hline
\end{tabular}

Span $1.6 \pm 0.04^{\circ} \quad 1.6 \pm 0.0^{*} \quad 1.1 \pm 0.1^{\mathrm{B}}$

Abbreviations: "LD: laser diffraction, 220 IA: $2 D$ dynamic image analysis, 330 R: 30 reconstruction

Fig. 2 Size distributions obtained using laser diffraction (dotted lines), 2D dynamic image analysis (dashed lines) and 3D reconstruction (solid line). The tables give the diameters and descriptive parameters corresponding to the different size distributions. The table on the left shows data for the FP3 microgels (A) and the table on the right shows data for the P4.5 microgels (B). Values with different letters in the same row differ significantly at $\mathrm{p}<0.05$.

Although unimodal and in the same size ranges (similar order of magnitude), the distributions obtained also showed some differences depending on the measurement technique used, mainly for bigger sizes. For the FP3 stirred yogurt (Fig. 2 A), the LD measurement displayed the broadest distribution resulting in a significantly higher span. The size distributions obtained from 2D image analysis and 3D reconstruction had similar spans, but the 2D sizes were significantly bigger $(\mathrm{d}(0.5), \mathrm{d}(0.9), \mathrm{D}[4,3])$. For the P4.5 stirred yogurt (Fig. 2 B), the 3D distribution differed from that of the LD and 2D distributions, in particular by being significantly narrower (smaller span) and by displaying fewer big microgels (smaller $\mathrm{d}(0.9)$ and 
$\mathrm{D}[4,3])$. Several authors also reported that the size distributions differed with the technique used when the particles were non-spherical particles. Yu \& Hancock (2008) showed that the LD size distributions of elongated microcrystalline cellulose particles $(150-250 \mu \mathrm{m})$ were wider than their 2D distributions measured by dynamic image analysis. Califice et al. (2013) demonstrated that 2D dynamic image analysis tended to overestimate/underestimate the size of non-spherical particles $(50-500 \mu \mathrm{m}$ elongated metallic particles) compared to $3 \mathrm{D}$ reconstruction values obtained from X-ray microtomography images. The literature explained the differences in size distributions by both the measurement technique and the method of calculation used (Califice et al., 2013; Köhler et al., 2008; Tinke et al., 2008; Yu \& Hancock, 2008). In the present study, LD hypothesized that the particles analyzed were spherical. The calculation of the 2D diameter corresponded to the diameter of a circle of equal projection area (EQPC) and depended on the orientation of the microgel when measured. With 3D reconstruction, the measurement was protein-specific (CLSM staining) and the calculated diameter corresponded to the diameter of the equivalent sphere in volume (and did not depend on the orientation of the microgel). All these differences between the techniques likely explain the slight discrepancies shown in Fig. 2 for each of the stirred yogurts and suggest their microgels were not spherical. Further analysis of the microgel shape was thus performed to better understand the differences in size distribution, to compare the techniques and to characterize the stirred yogurt microgels more precisely.

\subsection{Comparison of the shape of the microgels $(2 \mathrm{D}, 3 \mathrm{D})$}

2D image analysis and 3D reconstruction were both used to determine the microgel shape. Fig. $3 \mathrm{~A}$ illustrates how the characteristic lengths were obtained from 2D $\left(F_{\max }\right.$ and $\left.F_{\min }\right)$ and 3D (major and minor) analyses. Fig. $3 \mathrm{~B}(a)$ and $\mathrm{C}(a)$ below show the distributions of the different lengths for FP3 and P4.5 stirred yogurts, respectively. These length distributions are classically used to provide information about the shape (spherical or elongated) of the particles (Califice et al., 2013; Yu \& Hancock, 2008). When microgels are spherical, the maximum length is obviously the same as the minimum length (Yu \& Hancock, 2008). Here, minor and $F_{\min }$ length distributions were smaller than major and $F_{\max }$ distributions for FP3 and P4.5, indicating that stirred yogurt microgels are not spherical, as previously suspected based on differences in size distributions obtained with the LD, 2D dynamic image analysis and 3D reconstruction. These results are in agreement with the fresh cheese microgels observed by Hahn et al. (2014) using CLSM, which were also irregular in shape. In addition, the P4.5 length distributions obtained from the 2D image analysis were broader than those obtained from 3D reconstruction. The differences between the 3D lengths (i.e. between minor and major) were more important than the differences between the $2 \mathrm{D}$ lengths (i.e. between $F_{\min }$ and $\left.F_{\max }\right)$. These results reveal some differences between the 2D and 3D distributions that can mainly be explained by the way the lengths were obtained with each technique (Fig. 3 A). From the 2D image analysis, $F_{\min }$ and $F_{\max }$ lengths could be biased by the orientation of microgels when measured (orientated lengthwise due to the flow). A similar concern has been expressed for irregular concrete aggregates (Cepuritis, Garboczi, Jacobsen, \& Snyder, 2017). With 3D reconstruction, the microgel may not be in direct contact with the ellipsoid edge to encompass the entire microgel (in length, width and thickness) (Fig. 3 (A)). This 
technique may therefore overestimate the minor and major lengths. Based on X-ray microcomputed tomography, Cepuritis et al. (2017) reported that 3D minor and major lengths depended on the dimension of the rectangular box enclosing the particle. In addition, in the present study, there were more differences between the two techniques for the P4.5 stirred yogurt. This result showed that P4.5 stirred yogurt microgels are more heterogeneous in shape (with more different types of elongation) than FP3 ones.

Images $(b)$ and $(c)$ in Fig. $3 \mathrm{~B}$ and $\mathrm{C}$, show the stirred yogurt microgels obtained using 3D reconstruction (from the z-stack confocal images) and 2D images analysis, respectively, confirming that the microgels were very heterogeneous in size and shape. This is in agreement with the results of Hahn et al. (2015), who observed CLSM images of fresh cheese under different processing conditions. 2D images of FP3 and P4.5 stirred yogurts also showed different degrees of microgel compactness (Fig. 3 (c)). For example, the enlarged \#1 microgels obtained from the screenshots (2D image analysis) appear to be more compact than the \#2 ones (Fig. 3 B (c) and Fig. 3 C (c)).
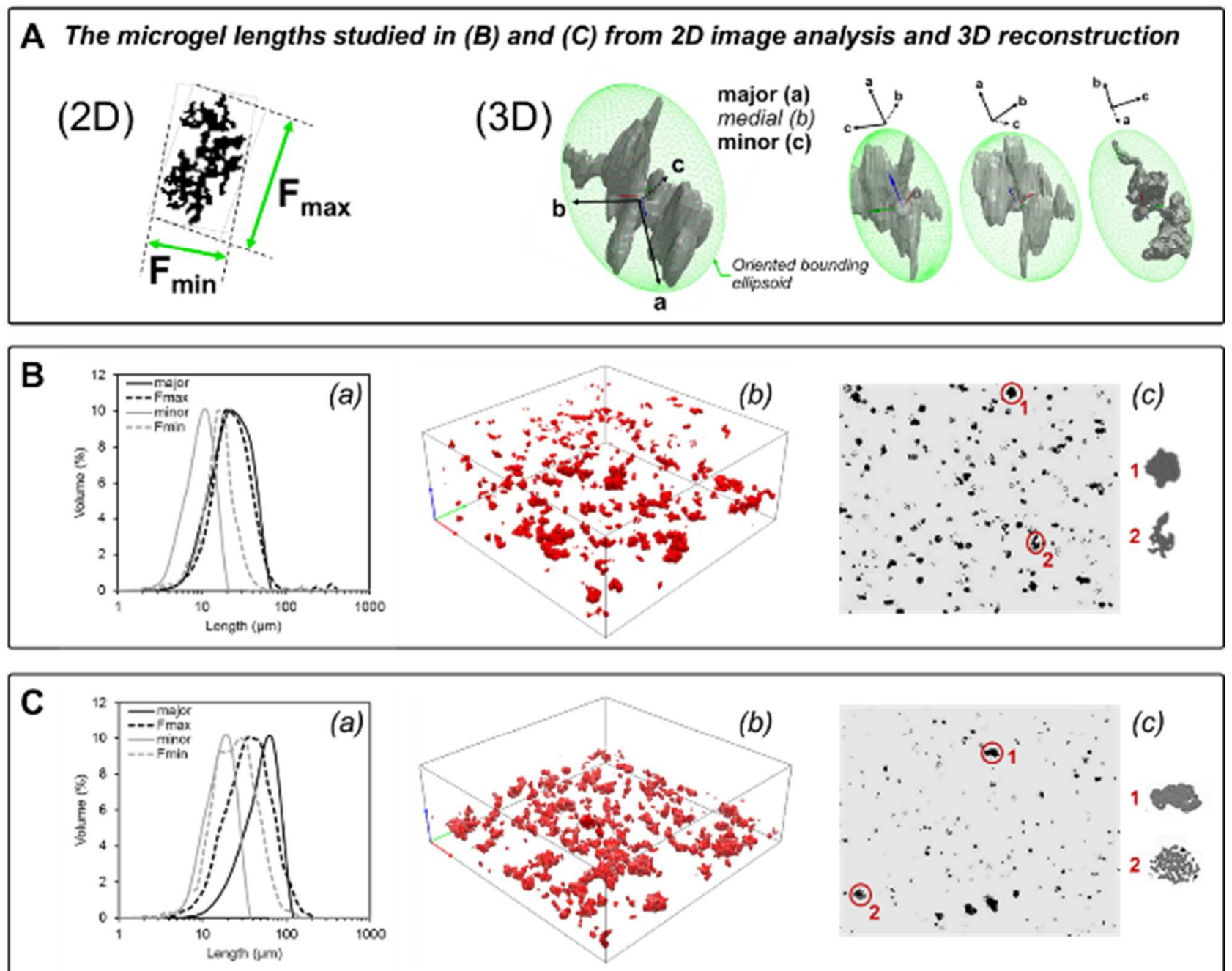

Fig. 3 Details of microgel lengths (major, Fmax, minor, Fmin) (A) studied for FP3 (B) and P4.5 (C) stirred yogurts through: (a) the length distributions (average curves) obtained from 2D image analysis (dashed lines) and 3D reconstruction (solid lines), (b) the $3 \mathrm{D}$ reconstructions and (c) a screenshot of the movie processed throughout 2D image analysis. 
Fig. 4 presents the roughness index distributions obtained for FP3 (A) and P4.5 (B) stirred yogurts. The roughness index value ranges from 0 to 1 and describes the surface unevenness on the microgels. The index tends towards 1 for microgels with no unevenness (i.e. a smooth circle (2D) or sphere (3D)).

For each technique considered independently, the roughness distributions of the two stirred yogurts were globally similar, even if that of P4.5 was slightly broader. The differences in the yogurt compositions and stirring processes could explain this slight difference in roughness distributions. However, there were bigger differences between the $2 \mathrm{D}$ and $3 \mathrm{D}$ roughness distributions. Using $3 \mathrm{D}$ reconstruction, the distributions were narrow and unimodal, with a median roughness of 0.8 , whereas using $2 \mathrm{D}$ images analysis, they displayed a main peak with a shoulder, with a first peak at 0.6 and a second one at $0.8-0.9$. The 2D distributions were also broader (from 0.2-0.3 to 1 ) than $3 \mathrm{D}$ ones (0.4-0.5 to 1). These results indicate that the stirred yogurt microgels appears less uniform in roughness with 2D images analysis. The difference could be explained by the processing steps used to reconstitute the 3D microgels. The application of a median filter and the selection of a threshold (subsection 2.4.1 and Fig. 1) could smooth the microgel surfaces (i.e. the boundary between the background and the microgels) and therefore underestimate the width of the roughness distributions.

Although these microgels tended towards a smooth surface (roughness mostly between 0.7 and 0.8 ), the range of widths of the distribution underlined the heterogeneity of the stirred yogurt microgels that can be linked to microgel size. Some studies already linked the shape of the particles such as the roughness index, to their size (Yan \& Shi, 2014; Zhou \& Wang, 2017). In the present study, the roughness index decreased (i.e. surface unevenness was greater) in bigger microgels (data not shown). Rougher microgels are probably due to the bigger size $(>30 \mu \mathrm{m})$ of microgels that were mostly measured using $2 \mathrm{D}$ analysis rather than $3 \mathrm{D}$ reconstruction (subsection 3.1).
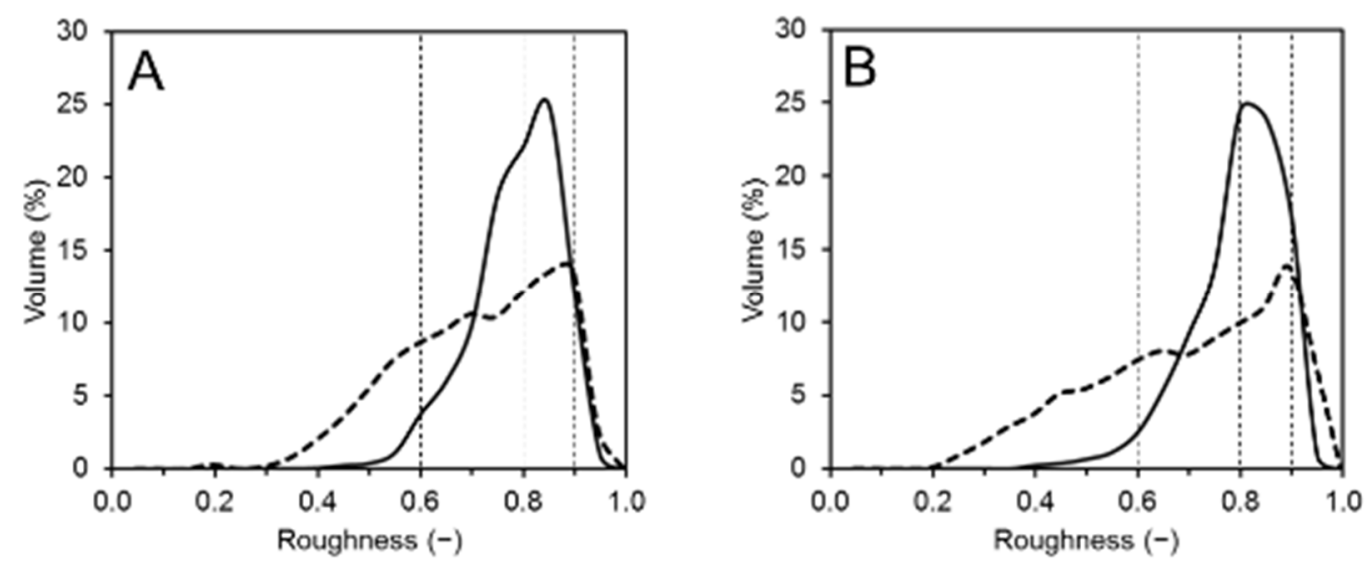

Fig. 4 Weighted average curves (three repetitions) of the roughness index distributions obtained from 2D image analysis (dashed lines) and 3D reconstruction (solid lines) for FP3 (A) and P4.5 (B) stirred yogurts.

\subsection{Comparison of the microgel fractal dimension (LD, 2D, 3D)}

All three techniques were used to estimate the microgel fractal dimension of the two stirred yogurts (FP3 and P4.5). For the LD measurements, the slopes were extracted with very high regression coefficients 
systematically higher than 0.96 . With 2D dynamic image analysis, the two-dimensional fractal dimensions $\left(D_{2}\right)$ were also extracted with very high regression coefficients, i.e. higher than 0.91 . These high values indicated excellent fitting, thus allowing high confidence in the results obtained using these techniques (Table 1).

Table 1 Average fractal dimensions obtained from laser diffraction, 2D image analysis and 3D reconstruction. Values with different letters in the same column differ significantly at $p<0.05$.

\begin{tabular}{ccc}
\hline Technique & FP3 & P4.5 \\
\hline Laser diffraction & $2.31 \pm 0.01 a$ & $2.37 \pm 0.03 a$ \\
\hline 2D image analysis & $2.05 \pm 0.02 c$ & $2.08 \pm 0.02 c$ \\
\hline 3D reconstruction & $2.26 \pm 0.01 b$ & $2.27 \pm 0.03 b$ \\
\hline
\end{tabular}

352

The average of the mass fractal dimensions $\left(D_{f}\right)$ obtained from LD and 3D reconstruction were similar even if significantly different, with values around 2.3 for the two samples. These values are in good agreement with values reported in the literature for fermented stirred milk gels (van Marle et al., 1999). In conventional studies on Brownian aggregation of particles (generally latex suspensions), the obtained $D_{f}$ values are discussed in the frame of two limiting regimes. Such studies are conducted under low volume concentrations during aggregation (typically $10^{-3}-10^{-4} \%$ ), ensuring the validity of the theory. When there is no energy barrier between the colliding particles, each collision leads to aggregation. This regime is called "diffusion limited aggregation" (DLA) and results in loose open structures with $D_{f}$ around $1.7-1.8$. When the repulsion forces are still significant, the particles can penetrate the aggregate structure before adhering. This regime is called "reaction limited aggregation" (RLA) and leads to denser aggregates, with $D_{f}$ around 2.1. In the present study, the volume fractions of milk proteins (before any dilution) were higher than $1 \%$ in both stirred yogurts (FP3 and P4.5), which explains why the values obtained were significantly higher (Bremer, van Vliet, \& Walstra, 1989). In addition, the colloidal calcium phosphate, which ensures the structure integrity of the casein micelles, dissolves during acidification. This dissolution results in the loosening of the micelles (increasing their volume), which likely promotes the compaction of the protein aggregates due to loss of repulsive interactions and thus leads to denser structures (Andoyo et al., 2015).

The $D_{f}$ value calculated from 3D reconstruction could be considered as the most accurate of the three techniques, because it relies on direct visualization of the aggregates and assumes no strong assumption. However, the LD technique proved to be a very good alternative technique to obtain $D_{f}$ as the differences between LD and 3D were very small. However, the values obtained using 2D image analysis and the equation proposed by Lee \& Kramer (2004) differed more from 3D measurements. Estimating $D_{f}$ from 2D image analysis using this equation thus appears to be questionable in the case of stirred yogurt microgels. Lee \& Kramer (2004) reported underestimation of $D_{f}$ in the case of E. coli aggregates and explained that it was partly because E. coli were not spherical, which could also be the case of the stirred yogurt microgels. The $3 \mathrm{D}$ and $\mathrm{LD}$ techniques are thus recommended over $2 \mathrm{D}$ analysis. 


\subsection{Comparison of the advantages and limitations of $L D, 2 D$ and $3 D$}

To complete the comparison of the performances of the three techniques, Table 2 summarizes the size distribution range, the measurement conditions, the time needed for measurement and data treatment per sample, the properties obtained directly or calculated from the data as well as the assumptions and weaknesses.

While LD, 2D image analysis and 3D reconstruction proved to be quite consistent in characterizing stirred yogurt microgels, Table 2 shows that they each had their advantages and limitations. The LD technique mainly assumes that the analyzed particles are homogeneous and spherical, which has been shown (subsection 3.1) to lead to overestimation of the bigger particles and/or underestimation of the smaller particles when measuring the microgel sizes of the stirred yogurts. Moreover, this technique requires refractive and adsorption indexes, which can be difficult to estimate for complex systems composed of different ingredients. However, in the case of the stirred yogurt microgels, these indexes were not problematic since no variation in the size distribution was observed when their values varied (due to the sufficiently large size of the microgels). Although LD obviously does not allow access to shape factors, it is quick and user-friendly for accessing the size distribution and the fractal dimension. It also makes it possible to measure particles less than a micron in size, which is not the case of the $2 \mathrm{D}$ and $3 \mathrm{D}$ techniques presented here (limited by their optical geometry characteristic).

Table 2 Comparison of laser diffraction, 2D image analysis and 3D reconstruction. Information in bold indicates the advantages of each technique.

\begin{tabular}{|c|c|c|c|}
\hline Technique & Laser diffraction & 2D image analysis & $3 \mathrm{D}$ reconstruction \\
\hline Equipment & $\begin{array}{l}\text { MasterSizer } 2000 \\
\text { (Malvern) }\end{array}$ & $\begin{array}{l}\text { QICPIC/R and LIXELL } \\
\text { (Sympatec) }\end{array}$ & $\begin{array}{l}\text { CLSM (Leica) and Scan IP } \text { IP }^{\mathrm{TM}} \\
\text { (Simpleware) }\end{array}$ \\
\hline Size range & 0.02 to $2,000 \mu \mathrm{m}$ & 1 to $750 \mu \mathrm{m}$ (M4 lens) & $\begin{array}{l}0.532 \mu \mathrm{m}(\text { i.e. pixel) to } \\
\text { a few millimeters }\end{array}$ \\
\hline $\begin{array}{l}\text { Measurement } \\
\text { conditions }\end{array}$ & $\begin{array}{c}\text { Dilution 1:100, Agitation, } \\
\text { Pumping }\end{array}$ & $\begin{array}{c}\text { Dilution 1:2000, Agitation, } \\
\text { Pumping } \\
\end{array}$ & Dilution 1:100, Staining \\
\hline $\begin{array}{l}\text { Measuring time per } \\
\text { sample }\end{array}$ & $10 \mathrm{~min}$ & $30 \mathrm{sec}$ & $30 \mathrm{~min}$ \\
\hline $\begin{array}{l}\text { Time needed for data } \\
\text { treatment per sample }\end{array}$ & $10 \mathrm{~min}$ & $30 \mathrm{~min}$ & $1 \mathrm{~h}$ \\
\hline $\begin{array}{l}\text { Properties obtained } \\
\text { directly }\end{array}$ & Size & $\begin{array}{c}\text { Size, Shape factors, } \\
\text { Visualization of microgel } \\
\text { projection (2D) }\end{array}$ & - \\
\hline Calculated properties & Fractal dimension & Fractal dimension & $\begin{array}{c}\text { Size, Shape factors, Fractal } \\
\text { dimension, realistic } \\
\text { visualization of the microgels } \\
\text { (3D) }\end{array}$ \\
\hline $\begin{array}{l}\text { Assumptions and } \\
\text { limitations }\end{array}$ & $\begin{array}{l}\text { (i) Particles considered as } \\
\text { homogeneous and spherical } \\
\text { (ii) Need for refractive and } \\
\text { absorption indexes } \\
\text { (iii) No access to particle shape }\end{array}$ & $\begin{array}{l}\text { (i) Data based on projected } \\
\text { areas of the particles } \\
\text { (depending on their orientation) } \\
\text { (ii) Low camera resolution } \\
\text { (iii) Need for low } \\
\text { concentrations of particles }\end{array}$ & $\begin{array}{l}\text { (i) Threshold to select pixels of } \\
\text { interest (identification of the } \\
\text { stained particles) } \\
\text { (ii) Small number of particles } \\
\text { (iii) Time consuming data } \\
\text { processing }\end{array}$ \\
\hline
\end{tabular}


(colloidal systems, for example). The first limitation is that although the mass fractal dimension can be estimated using a specific relation reported in the literature, (subsection 3.3) its use was shown to be questionable in the case of the stirred yogurt microgels. Moreover, the time required to process the data is quite long, and this technique analyzes the projected areas (2D) of the measured particles, which may depend on their orientation during measurement. On the other hand, it has the advantage of allowing a very large number of particles to be analyzed, which should offsets the orientation bias. Moreover, it enables relatively rapid measurement and direct access to the size and shape properties. It provided a $2 \mathrm{D}$ view of the particles that revealed that the microgels were not spherical, but showed varying degrees of roughness, and were sometimes porous (fractal dimension) in stirred yogurt.

The smallest size that can be measured with $3 \mathrm{D}$ reconstruction depends on the resolution of the microscope and may be high (i.e. allowing to observe small sizes) in food structure analysis. Data acquisition is time consuming and the analysis of the properties of size, shape and fractal dimension requires complete data processing. Moreover, a threshold has to be chosen to select pixels of interest (identification of the stained microgels). The results showed in subsections 3.1, 3.3 and 3.3 demonstrated that the choice made for this study was appropriate in the case of the stirred yogurt microgels studied here. One of the advantages of the 3D technique (using CLSM) is the limited shear undergone by the particles. This is particularly relevant for the study of brittle systems such as stirred yogurt microgels and most food matrices. Based on molecule staining, it also allows the selection of specific compounds within the particles and tailored measurement of the structure. The main strength of $3 \mathrm{D}$ reconstruction is that it enables full visualization of the particles, with no orientation bias or sphericity assumption. This specificity was particularly useful in the present study since it offered the opportunity to clearly observe the diverse sizes and shapes of the yogurt microgels.

\section{Conclusions}

Laser diffraction, 2D dynamic image analysis and 3D reconstruction were shown to be relevant and complementary for the characterization of the size (through PSD), shape and fractal dimension of heterogeneous (in composition) and irregularly shaped systems like stirred yogurt microgels. By comparing LD with 2D image analysis and 3D reconstruction on two different stirred yogurts, we showed that LD was fully relevant to access the size distribution and the mean mass fractal dimension of non-spherical yogurt microgels. The use of 2D dynamic image analysis and 3D reconstruction also raised the question of the characterization of the shape of the stirred yogurt microgels. While rarely used for food systems, 2D dynamic image analysis proved to be advantageous to visualize the microgels and quickly estimate their morphological parameters. 3D reconstruction also has very useful features as it enables access to shape factors while avoiding the possible bias resulting from particle orientation using $2 \mathrm{D}$ analysis. However, the 3D technique usually entails time consuming sample preparation and analysis, and is thus not really to be recommended for routine analysis. This comparison of the three techniques provides useful guidelines for studying complex food systems. Moreover, these techniques can offer new perspectives to accurately explain the relationship between the microstructure and the macro-scale properties (such as flow properties) of a food system at each step of its processing chain. 
438

\section{Supplementary material}

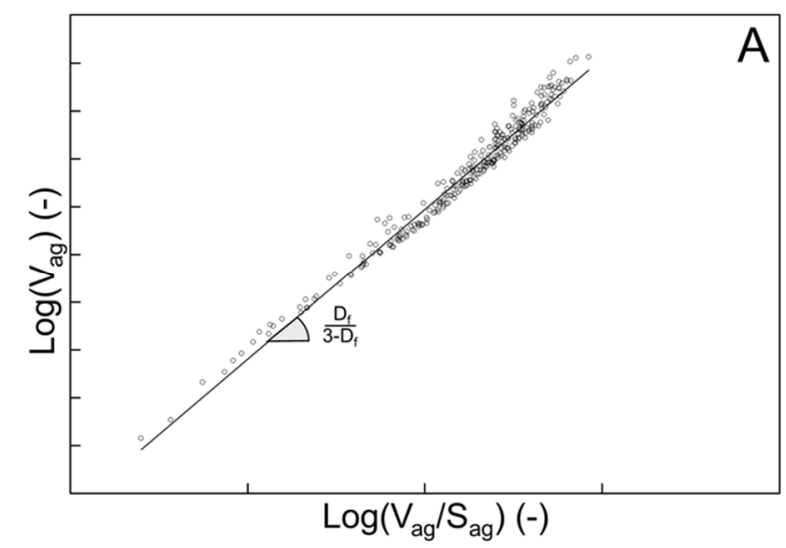



Fig. 5 Graph of principle used to obtain the fractal dimension from the laser diffraction data (A) and the 3D reconstruction data $(\mathbf{B})$ where $q\left(\mathrm{~m}^{-1}\right)$ is the scattering vector, $I(q)$ is the light scattered intensity, $V_{a g}\left(\mathrm{~m}^{3}\right)$ is the volume and $S_{a g}\left(\mathrm{~m}^{2}\right)$ the surface envelope of each aggregate.

\section{References}

Andoyo, R., Guyomarc, F., \& Burel, A. (2015). Spatial arrangement of casein micelles and whey protein aggregate in acid gels: Insight on mechanisms. Food Hydrocolloids, 51, 118-128. https://doi.org/10.1016/j.foodhyd.2015.04.031

Baalousha, M., Manciulea, A., Cumberland, S., Kendall, K., \& Lead, J. R. (2008). Aggregation and surface properties of iron oxide nanoparticles: influence of $\mathrm{pH}$ and natural organic matter. Environmental Toxicology and Chemistry / SETAC, 27(9), 1875-82. https://doi.org/10.1897/07-559.1

Bremer, L. G. B., van Vliet, T., \& Walstra, P. (1989). Theoretical and experimental study of the fractal nature of the structure of casein gels. Journal of the Chemical Society, Faraday Transactions 1: Physical Chemistry in Condensed Phases, 85(10), 3359-3372. https://doi.org/10.1039/F19898503359

Burns, J. L., Yan, Y., Jameson, G. J., \& Biggs, S. (1997). A light scattering study of the fractal aggregation behavior of a model colloidal system. Langmuir, 13(24), 6413-6420. Retrieved from http://pubs.acs.org/doi/abs/10.1021/la970303f

Bushell, G. C., Yan, Y. D., Woodfield, D., Raper, J., \& Amal, R. (2002). On techniques for the measurement of the mass fractal dimension of aggregates. Advances in Colloid and Interface Science, 95(1), 1-50. https://doi.org/10.1016/S0001-8686(00)00078-6

Califice, A., Michel, F., Dislaire, G., \& Pirard, E. (2013). Influence of particle shape on size distribution measurements by 3D and 2D image analyses and laser diffraction. Powder Technology, 237, 67-75. https://doi.org/10.1016/j.powtec.2013.01.003 
Carugo, D., Ankrett, D. N., Zhao, X., Zhang, X., Hill, M., O’Byrne, V., ... Lewis, A. L. (2015). Benefits of polidocanol endovenous microfoam (Varithena $\left.{ }^{\circledR}\right)$ compared with physician-compounded foams. Phlebology, 31(4), 283-295. https://doi.org/10.1177/0268355515589063

Cayot, P., Schenker, F., Houzé, G., Sulmont-Rossé, C., \& Colas, B. (2008). Creaminess in relation to consistency and particle size in stirred fat-free yogurt. International Dairy Journal, 18(3), 303-311. https://doi.org/10.1016/j.idairyj.2007.06.009

Cepuritis, R., Garboczi, E. J., Jacobsen, S., \& Snyder, K. A. (2017). Comparison of 2-D and 3-D shape analysis of concrete aggregate fines from VSI crushing. Powder Technology, 309, 110-125. https://doi.org/10.1016/j.powtec.2016.12.037

Chardot, V., Banon, S., Misiuwianiec, M., \& Hardy, J. (2002). Growth kinetics and fractal dimensions of casein particles during acidification. Journal of Dairy Science, 85(1), 8-14. https://doi.org/10.3168/jds.S0022-0302(02)74046-0

Chung, C., Degner, B., \& Julian, D. (2014). Development of Reduced-calorie foods : Microparticulated whey proteins as fat mimetics in semi-solid food emulsions. Food Research International, 56, $136-145$. https://doi.org/10.1016/j.foodres.2013.11.034

Forrest, S. R., \& Witten, T. A. (1979). Long-range correlations in smoke-particle aggregates. Journal of Physics A: Mathematical and General, 12(5), L109--L117. https://doi.org/10.1088/0305-4470/12/5/008

Gregory, J. (2009). Monitoring particle aggregation processes. Advances in Colloid and Interface Science, 147-148, 109-123. Retrieved from http://linkinghub.elsevier.com/retrieve/pii/S0001868608001462

Hahn, C., Müller, E., Wille, S., Weiss, J., Atamer, Z., \& Hinrichs, J. (2014). Control of microgel particle growth in fresh cheese (concentrated fermented milk) with an exopolysaccharide-producing starter culture. International Dairy Journal, 36(1), 46-54. https://doi.org/10.1016/j.idairyj.2013.12.011

Hahn, C., Nöbel, S., Maisch, R., Rösingh, W., Weiss, J., \& Hinrichs, J. (2015). Adjusting rheological properties of concentrated microgel suspensions by particle size distribution. Food Hydrocolloids, 49, 183-191. https://doi.org/10.1016/j.foodhyd.2015.03.020

Hahn, C., Sramek, M., Nöbel, S., \& Hinrichs, J. (2012). Post-processing of concentrated fermented milk: Influence of temperature and holding time on the formation of particle clusters. Dairy Science and Technology, 92(1), 91-107. https://doi.org/10.1007/s13594-011-0046-1

Hentschel, M. L., \& Page, N. W. (2003). Selection of descriptors for particle shape characterization. Particle and Particle Systems Characterization, 20(1), 25-38. https://doi.org/10.1002/ppsc.200390002

Huc, D., Michon, C., Bedoussac, C., \& Bosc, V. (2016). Design of a multi-scale texture study of yoghurts using rheology, and tribology mimicking the eating process and microstructure characterisation. International Dairy Journal, 61, 126-134. https://doi.org/10.1016/j.idairyj.2016.05.003

Jiang, Q., \& Logan, B. E. (1991). Fractal Dimensions of Aggregates Determined from Steady-State Size Distributions. Environmental Science and Technology, 205(12), 2031-2038. https://doi.org/10.1021/es00024a007

Köhler, U., Stübinger, T., List, J., \& Witt, W. (2008). Investigations on non-Spherical Reference Material Using Laser Diffraction and Dynamic Image Analysis. Particulate systems analysis.

Lachin, K., Le Sauze, N., Di Miceli Raimondi, N., Aubin, J., Gourdon, C., \& Cabassud, M. (2017). Aggregation and breakup of acrylic latex particles inside millimetric scale reactors. Chemical Engineering and Processing: Process Intensification, 113, 65-73. https://doi.org/10.1016/j.cep.2016.09.021

Lazzari, S., Nicoud, L., Jaquet, B., Lattuada, M., \& Morbidelli, M. (2016). Fractal-like structures in colloid science. Advances in Colloid and Interface Science, 235, 1-13. https://doi.org/10.1016/j.cis.2016.05.002

Lee, C., \& Kramer, T. A. (2004). Prediction of three-dimensional fractal dimensions using the twodimensional properties of fractal aggregates. Advances in Colloid and Interface Science, 112(1-3), 4957. https://doi.org/10.1016/j.cis.2004.07.001

Lee, W.-J., \& Lucey, J. A. (2006). Impact of Gelation Conditions and Structural Breakdown on the Physical 
and Sensory Properties of Stirred Yogurts. Journal of Dairy Science, 89(7), 2374-2385. https://doi.org/10.3168/jds.S0022-0302(06)72310-4

Leverrier, C., Moulin, G., Cuvelier, G., \& Almeida, G. (2017). Assessment of deformability of soft plant cells by 3D imaging. Food Structure, 14(September), 95-103. https://doi.org/10.1016/j.foostr.2017.07.002

List, J., Köhler, U., Witt, W., Gmbh, S., \& Pulverhaus, A. (2011). Dynamic Image Analysis extended to Fine and Coarse Particles. Particulate Systems Analysis, 1-5.

Mallipeddi, R., Saripella, K. K., \& Neau, S. H. (2014). Use of fine particle ethylcellulose as the diluent in the production of pellets by extrusion-spheronization. Saudi Pharmaceutical Journal, 22(4), 360-372. https://doi.org/10.1016/j.jsps.2013.11.001

Malvern Instruments Ltd. (2007). Mastersizer 2000 - User Manual - MAN0384 Issue 1.0.

Mandelbrot, B. (1975). Les objets fractals, forme, hasard et dimension. Paris: Flammarion.

Mellema, M., Walstra, P., van Opheusden, J. H. J., \& van Vliet, T. (2002). Effects of structural rearrangements on the rheology of rennet- induced casein particle gels. Advances in Colloid and Interface Science, 98(1), 25-50.

Mokoonlall, A., Nöbel, S., \& Hinrichs, J. (2016). Post-processing of fermented milk to stirred products: Reviewing the effects on gel structure. Trends in Food Science and Technology, 54, 26-36. https://doi.org/10.1016/j.tifs.2016.05.012

Mortazavian, A. M., Rezaei, K., \& Sohrabvandi, S. (2009). Application of advanced instrumental methods for yogurt analysis. Critical Reviews in Food Science and Nutrition, 49(2), 153-163. https://doi.org/10.1080/10408390701764807

Nöbel, S., Ross, N. L., Protte, K., Körzendörfer, A., Hitzmann, B., \& Hinrichs, J. (2016). Microgel particle formation in yogurt as influenced by sonication during fermentation. Journal of Food Engineering, 180, 29-38. https://doi.org/10.1016/j.jfoodeng.2016.01.033

Panouillé, M., Durand, D., Nicolai, T., Larquet, E., \& Boisset, N. (2005). Aggregation and gelation of micellar casein particles. Journal of Colloid and Interface Science, 287(1), 85-93. https://doi.org/10.1016/j.jcis.2005.02.008

Perez, M., Décaudin, B., Maiguy-Foinard, A., Barthélémy, C., Lebuffe, G., Storme, L., \& Odou, P. (2017). Dynamic Image Analysis to Evaluate Subvisible Particles during Continuous Drug Infusion in a Neonatal Intensive Care Unit. Scientific Reports, 7(1), 1-8. https://doi.org/10.1038/s41598-017-10073$\mathrm{y}$

Podczek, F. (1997). A shape factor to assess the shape of particles using image analysis. Powder Technology, 93, 47-53.

Raper, J. A., \& Amal, R. (1993). Measurement of aggregate fractal dimensions using static light scattering. Particle and Particle Systems Characterization, 10(5), 239-245. Retrieved from http://onlinelibrary.wiley.com/doi/10.1002/ppsc.19930100505/abstract\%5Cnpapers2://publication/uuid/ D7EC2CC6-7FAD-4820-A345-F5985D21E4C4

Selomulya, C., Amal, R., Bushell, G., \& Waite, T. D. (2001). Evidence of shear rate dependence on restructuring and breakup of latex aggregates. Journal of Colloid and Interface Science, 236(1), 67-77.

Serra, T., \& Casamitjana, X. (1998). Structure of the aggregates during the process of aggregation and breakup under a shear flow. Journal of Colloid and Interface Science, 206(2), 505-511. https://doi.org/10.1006/jcis.1998.5714

Shewan, H. M., \& Stokes, J. R. (2013). Review of techniques to manufacture micro-hydrogel particles for the food industry and their applications. Journal of Food Engineering, 119(4), 781-792. https://doi.org/10.1016/j.jfoodeng.2013.06.046

Sodini, I., Remeuf, F., Haddad, S., \& Corrieu, G. (2004). The Relative Effect of Milk Base, Starter, and Process on Yogurt Texture: A Review. Critical Reviews in Food Science and Nutrition, 44, 113-137. https://doi.org/10.1080/10408690490424793

Sorensen, C. M. (2001). Light scattering by fractal aggregates : A review. Aerosol Science and Technology, 
35(2), 648-687.

Tinke, A. P., Carnicer, A., Govoreanu, R., Scheltjens, G., Lauwerysen, L., Mertens, N., ... Brewster, M. E. (2008). Particle shape and orientation in laser diffraction and static image analysis size distribution analysis of micrometer sized rectangular particles. Powder Technology, 186(2), 154-167. https://doi.org/10.1016/j.powtec.2007.11.017

Torres, I. C., Amigo Rubio, J. M., \& Ipsen, R. (2012). Using fractal image analysis to characterize microstructure of low-fat stirred yoghurt manufactured with microparticulated whey protein. Journal of Food Engineering, 109(4), 721-729. https://doi.org/10.1016/j.jfoodeng.2011.11.016

van den Berg, L., Jan Klok, H., van Vliet, T., van der Linden, E., van Boekel, M. A. J. S., \& van de Velde, F. (2008). Quantification of a 3D structural evolution of food composites under large deformations using microrheology. Food Hydrocolloids, 22(8), 1574-1583. https://doi.org/10.1016/j.foodhyd.2007.11.002

Van Marle, M. (1998). Structure and rheological properties of yogurt gels and stirred yogurt. University of Twente Netherlands.

van Marle, M. E., van den Ende, D., de Kruif, C. G., \& Mellema, J. (1999). Steady-shear viscosity of stirred yogurts with varying ropiness. Journal of Rheology, 43(6), 1643-1662. https://doi.org/10.1122/1.551065

Vétier, N., Banon, S., Chardot, V., \& Hardy, J. (2003). Effect of Temperature and Aggregation Rate on the Fractal Dimension of Renneted Casein Aggregates. Journal of Dairy Science, 86(8), 2504-2507. https://doi.org/10.3168/jds.S0022-0302(03)73844-2

Yan, W. M., \& Shi, Y. (2014). Evolution of grain grading and characteristics in repeatedly reconstituted assemblages subject to one-dimensional compression. Géotechnique Letters, 4(3), 223-229. https://doi.org/10.1680/geolett.14.00039

Yan, W. M., \& Su, D. (2017). Inferring 3D particle size and shape characteristics from projected 2D images: Lessons learned from ellipsoids. Computers and Geotechnics, (September), 0-1. https://doi.org/10.1016/j.compgeo.2017.11.015

Yu, W., \& Hancock, B. C. (2008). Evaluation of dynamic image analysis for characterizing pharmaceutical excipient particles. International Journal of Pharmaceutics, 361(1-2), 150-157. https://doi.org/10.1016/j.ijpharm.2008.05.025

Zhou, B., \& Wang, J. (2017). Generation of a realistic 3D sand assembly using X-ray micro-computed tomography and spherical harmonic-based principal component analysis. International Journal for Numerical and Analytical Methods in Geomechanics, 41(1), 93-109. https://doi.org/10.1002/nag.2548 